\title{
Current Status of Cancer Stem Cell Research
}

\section{Kereena Chukka*}

Department of Biotechnology, Acharya Nagarjuna University, India

*Corresponding author: Kereena Chukka, Department of Biotechnology, Acharya Nagarjuna university, Nagarjuna Nagar, Guntur, PIN 522510, India; Email: chukkakereena@gmail.com

\section{Editorial}

Volume 4 Issue 1

Received Date: February 12, 2020

Published Date: February 14, 2020

DOI: $10.23880 /$ jes- 16000137

\section{Editorial}

The purpose of this editorial is to highlight the recent Cancer Stem Cell research contributions and their approaches towards human cancers. Despite of major scientific and technological progress of research \& development for new therapeutic advances during the past decade, Cancer remains a significant public health problem. It's the second most common cause of death worldwide. In 2018, the global burden of disease estimated that a total of 18.1 million new cases and 9.6 million deaths were reported [1].

Cancer Stem Cells (CSCs) are a small sub-population of cancer cells found within tumors that can both self-renew by dividing and give rise to different types of cells that constitute the tumour. CSCs are tumorigenic, conceivably in contrast to other non-tumorigenic cancer cells; these cells might be responsible for the lack of success of therapeutic approaches currently available [2]. Single or combination of treatments target only the bulk of the tumor, and the elevated rates of tumor recurrences are attached to the accumulation of CSCs $[3,4]$.

The resistance mechanisms endowed by CSCs are attributed to several factors including a transient cellular quiescence, the accumulation of ATP-binding cassette transporters (ABC transporters) and disruption of cellular apoptosis [5]. Chemoresistance associated with CSCs is observed in colorectal, breast and ovarian cancers, where activation of the serine-threonine kinase named Aurora-A is involved in resistance to apoptosis and tumorigenicity maintenance [6]. There is an increasing evidence that CSCs are also distinctively resistant to radiation therapy, by stimulating the repair of DNA damaged, increasing activation of the DNA damage checkpoint, redistributing the cells in the cell cycle and reoxygenating and repopulating areas of hypoxia in the tumor.

CSCs are also responsible for cancer initiation, tumor relapse, and metastasis, emerging as the major target for the development of successful therapies. Administration of cisplatin is associated with accumulation of CSC and the chemoresistance is controlled by epigenetic modifications in salivary gland tumors $[7,8]$.

Current cancer treatments fail to cure metastatic tumors, even though they have cytostatic and/or cytotoxic effects on cancer cells, their ability to eliminate CSCs remains poorly understood. The knowledge attained on CSCs in recent years supports better detection, isolation and improved personalized treatment target of these cells [9]. As a result, the development of new combined therapies, including the use of immunotherapy, epigenetic modifiers, stemness inhibitors, and CSCs surface markers are currently in clinical trial [5].

Several approaches are being followed in order to target CSCs including- I) Antibodies and antibody-drug conjugates targeting surface receptors or circulating proteins including progastrin and leucine-rich repeat-containing G-protein coupled receptor 5 (LGR5); II) Small molecules targeting surface biomarkers or signaling pathways that maintain phenotypic features of CSCs such as Hedgehog/Wnt/Notch; III) Small molecules targeting the metabolic pathways involved in the maintenance of the CSCs phenotype such as stearoyl-CoA desaturase-1 (SCD1) or (3-hydroxy-3-methylglutaryl-coenzyme A reductase) HMG-CoA reductase. These approaches are a plethora of compounds that are being investigated at the exploratory level and/or are at different stages of preclinical or clinical development.

Molecular targets that control CSCs have the potential to drive the development of new drugs capable of destroying and preventing the accumulation of new CSC in cancer patients, which could prevent tumor relapse, metastasis, reducing toxicity and morbidity and ultimately improving 


\section{Journal of Embryology \& Stem Cell Research}

the cancer patient's outcomes. We expect to achieve a better understanding about the safety and efficacy of the combination approaches.

\section{References}

1. Ferlay J, Colombet M, Soerjomataram I, Mathers C, Parkin DM, et al. (2019) Estimating the global cancer incidence and mortality in 2018: GLOBOCAN sources and methods. Int J Cancer 144(8): 1941-1953.

2. Lin Y, Kong F, Li H, Xu D, Jia F, et al. (2018) Comparison of target volume and clinical effects of four radiotherapy plans for acute lymphoblastic leukemia prior to hematopoietic stem cell transplantation. Mol Med Rep 18(3): 2762-2770.

3. Szakacs G, Paterson JK, Ludwig JA, Booth-Genthe C, Gottesman MM (2006) Targeting multidrug resistance in cancer. Nature Reviews Drug Discovery. 5(3): 219-234.

4. Badve S, Nakshatri H (2012) Breast-cancer stem cellsbeyond semantics. The Lancet Oncology 13(1): e43-e48.
5. Talukdar S, Emdad L, Das SK, Sarkar D, Fisher PB (2016) Evolving strategies for therapeutically targeting cancer stem cells. Advances in Cancer Research 131: 159-191.

6. Cammareri P, Scopelliti A, Todaro M, Eterno V, Francescangeli F, et al. (2010) Aurora-a is essential for the tumorigenic capacity and chemoresistance of colorectal cancer stem cells. Cancer Research 70(11): 4655-4665.

7. Almeida LO, Guimaraes DM, Martins MD, Martins MA, Warner KA, et al. (2017) Unlocking the chromatin of adenoid cystic carcinomas using HDAC inhibitors sensitize cancer stem cells to cisplatin and induces tumor senescence. Stem Cell Res 21: 94-105.

8. Guimaraes DM, Almeida LO, Martins MD, Warenr KA, Silva RAS, et al. (2016) Sensitizing mucoepidermoid carcinomas to chemotherapy by targeted disruption of cancer stem cells. Oncotarget 7(27): 42447-42460.

9. Dawood S, Austin L, Cristofanilli M (2014) Cancer stem cells: implications for cancer therapy. Oncology 28(12): 1101-1107. 